\title{
Nicht aufgeben!
}

\section{Die Behandlung der NEURODERMITIS ist oft langwierig, Rückschläge dürfen Therapeut und Patient nicht verunsichern.}

Siegfried Kämper

Foto: (C) shutterstock_Roblan

AN NEURODERMITIS litt Traudel D.* bereits seit früher Kindheit. Die juckenden und nässenden Ekzeme zeigten sich damals zunächst nur an den Ellenbogen-Beugeseiten und Kniekehlen. Als sie dann in die Pubertät kam, traten Hauteffloreszenzen auch an ihren Oberschenkeln und Beinen auf. Weder Kinderarzt noch später Hausarzt sowie zahlreiche Hautärzte fanden die Ursache für die Neurodermitis. Allergietests blieben stets ohne Befund.

Nachdem Traudel D. sich als junge Frau hatte zur Krankenschwester ausbilden lassen und seither mehrmals täglich Handdesinfektionsmittel benutzte, waren auch ihre Hände bald von Neurodermitis gezeichnet. Die „offenen Hände“ waren auch der Grund dafür, dass sie mich im Spätjahr 2009 (5.10.2009) erstmals in meiner Praxis aufsuchte. Der damals 41-Jährigen drohte zu diesem Zeitpunkt aufgrund der Neurodermitis die Berufsunfähigkeit.

Schon auf den ersten Blick waren an Traudel D.'s Ellenbeugen und Kniekehlen die für die Neurodermitis typischen Hautzeichen zu sehen, besonders ausgeprägt waren die Hauteffloreszenzen an ihren Händen: Rötung, schorfiger Belag, Furchenbildung mit tiefen Einrissen, die Haut nässte zudem stark.
Bei der körperlichen Untersuchung fanden sich sonst keine weiteren Auffälligkeiten, außer an der Zunge. Die war zwar nicht belegt, die Schleimhaut jedoch blass, leicht geschwollen, und am Zungenrand fanden sich diskrete Zahnabdrücke. Ein Hinweis, dass die Leber der Patientin stark belastet war.

Ich führe bei allen Patienten standardmäßig eine Irisdiagnostik durch. Bei Traudel D. zeigte sich im Irisbild deutlich eine lymphatische Konstitution. Weitere Auffälligkeiten in der Iris bzw. im Auge waren keine zu sehen.

\section{Mitschuldig: Dysbiose im Darm}

Im Sinne einer ganzheitlichen Konstitutionsbehandlung verordnete ich der Patientin zunächst Badiaga Synergon 157 (Fa. Kattwiga), tgl. morgens 8 Tr., sowie Lymphaden ${ }^{\circledR}$ (Fa. Hevert), $3 \times \operatorname{tgl} .12$ Tr.

\footnotetext{
* Name von der Redaktion geändert
} 
Meiner Erfahrung nach liegt einer Neurodermitis immer eine Dysbiose der Darmflora zugrunde. Für die weitere Diagnostik übergab ich Traudel D. daher Versandmaterial für eine Stuhluntersuchung und erklärte ihr, wie eine Stuhlprobe korrekt zu entnehmen und zu versenden ist. Wir vereinbarten einen Folgetermin, um den Stuhlbefund zu besprechen und die daraus dann abzuleitende Medikation. Die topische Behandlung der Haut sollte Traudel D. wie gehabt fortführen und auch die Hautpflege wie gewohnt.

Am 27.10.2009 kam Traudel D. wieder in die Praxis, zwischenzeitlich lag mir der Befund der Stuhluntersuchung vor: Ein Defizit an Enterobakterien und physiologischen E. coli sowie eine Fehlbesiedelung mit Candida albicans im Darm waren nachgewiesen worden.

Ich verordnete Traudel D. zur Beseitigung des Candida albicans $3 \times$ tgl. 2 Kps. Adiclair ${ }^{\circledR}$ (Fa. Ardeypharm). Zum Ausgleich des Keimdefizits, zur Regeneration der Darmflora und zur Stimulierung der körpereigenen Abwehr erhielt die Patientin zudem $2 \times$ tgl. 1 Kps. Mutaflor ${ }^{\circledR}$ (Fa. Ardeypharm) sowie $3 \times \operatorname{tgl} 15$ Tr. ProSymbioflor ${ }^{\circledR}$ und $2 \times \operatorname{tgl} .20$ Tr. Symbioflor $1^{\circledR}$ (beide Fa. SymbioPharm), wobei mit der Einnahme des Symbioflor $1^{\circledR}$ noch 4 Tage gewartet werden sollte. Zusätzlich empfahl ich Traudel D., sich gemäß der „Antipilzdiät nach Prof. Dr. Rieth“ zu ernähren (siehe: http://www.hauss.de/index.php/downloads).

\section{Erstverschlimmerung war vorauszusehen}

Schon wenige Tage, nachdem die Patientin mit der Einnahme der Mittel begonnen hatte, „blühten“ die Ekzeme auf. Diese Reaktion, Folge einer Überschwemmung des Körpers mit Giftstoffen verursacht durch die Zerstörung der Candida-Besiedelung, ist meiner Erfahrung nach wesentlich weniger stark ausgeprägt, wenn vor der Behandlung mit Nystatin eine auf die Konstitution des Patienten abgestimmte Ausleitung erfolgt. Traudel D. hatte Lymphaden ${ }^{\circledR}$ erhalten, die Reaktion verlief daher eher mild.

Als Traudel D. mich am 10.11.2009 wieder in der Praxis aufsuchte, waren die Hauteffloreszenzen im Vergleich zum Ersttermin bereits weniger stark ausgeprägt. Die Behandlung mit $2 \times \operatorname{tgl}$. 20 Tr. Symbioflor $1^{\circledR}$ sollte weiter erfolgen. Sobald Adiclair ${ }^{\circledR}$ aufgebraucht war, sollte Traudel D. dann aber $2 \times \operatorname{tgl} .2$ Kps. Myrrhinil-Intest ${ }^{\circledR}$ (Fa. Repha) einnehmen, anstelle des Nystatin-Präparats. Der Grund: Für die weitere Behandlung war es wichtig zu wissen, wie stark der Darm noch mit Candida albicans besiedelt ist. Eine unter der Einnahme von Adiclair ${ }^{\circledR}$ durchgeführte Stuhluntersuchung würde das Ergebnis verfälschen. Um während der Einnahmepause eine erneute Zunahme der Candida-Besiedelung zu verhindern - es war davon auszugehen, dass der Darm nach wie vor von dem Hefepilz befallen war - erhielt Traudel D. das

\section{Nahrungsmittelunverträglichkeit und} Dysbiose der Darmflora sind fast immer an der Entstehung einer Neurodermitis beteiligt.

\section{KURZ GEFASST}

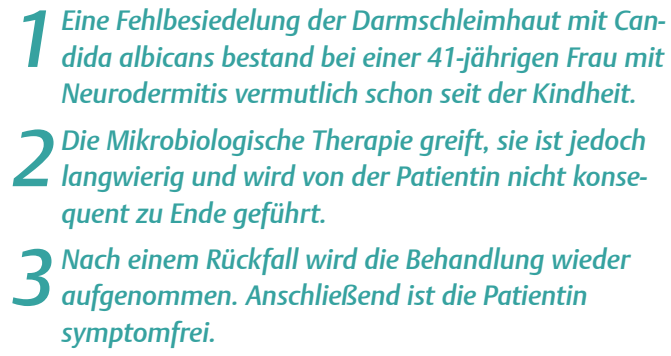

mykostatisch wirkende Myrrhinil-Intest ${ }^{\circledR}$, das den Befund nicht verfälscht. Ich übergab ihr erneut Versandmaterial für eine Stuhlanalyse, dieses Mal beschränkte ich den Untersuchungsauftrag jedoch auf den Nachweis von Candida albicans.

\section{Nahrungsmittelallergie ausschließen}

Am 8.12.2009 kam Traudel D. zur Besprechung des Laborbefundes in die Praxis. Candida albicans war noch immer nachweisbar, was meine Annahme bestätigte, dass die Infektion bereits vor sehr langer Zeit erfolgt war und die Besiedelung im Darm entsprechend lange bestand und massiv war. Ich verordnete der Patientin zusätzlich zu Myrrhinil-Intest ${ }^{\circledR}$ wieder $3 \times \operatorname{tgl} .2$ Kps. Adiclair ${ }^{\circledR}$. Im Sinne der isopathischen Therapie verordnete ich ihr zudem morgens 8 Tr. Albicansan D 5 (Fa. Sanum), eine aus Candida albicans hergestellte Nosode. Mutaflor ${ }^{\circledR}$ reduzierte ich auf $1 \mathrm{Kps}$. tgl. und verordnete zusätzlich $1 \times \operatorname{tgl} 1 / 2$ TL Synerga ${ }^{\circledR}$ (Fa. Laves). Das Präparat regeneriert die Schleimhaut und verbessert das Milieu, sodass unter diesen optimierten Bedingungen die Besiedelung der Darmflora mit lebenden physiologischen E. coli (Stamm Nissle) besser gelingen kann. Hinzu kommt, dass sich bei einer Nahrungsmittelunverträglichkeit Synerga ${ }^{\circledR}$ bestens bewährt hat, und nach meiner Erfahrung ist eine Nahrungsmittelunverträglichkeit häufig an der Entstehung der Neurodermitis beteiligt.

Zur Sensibilisierung gegenüber einem Nahrungsmittel kommt es u. a., wenn der Darm aufgrund emotionaler Belastungen (daher auch der Bezug im Namen „Neuro“) und/oder einer Dysbiose gereizt ist (Störfeld Darm). Auf der gereizten Schleimhautoberfläche werden Nahrungspartikel dann als Aggressor fehlinterpretiert, was eine Immunreaktion in Gang setzt, die sich häufig auch an der Haut zeigt. Liegt bei einem Neurodermitis-Patienten eine Nahrungsmittelunverträglichkeit vor, gilt es, für eine intakte Darmschleimhaut zu sorgen und das auslösende Nahrungsmittel zu meiden.

Während der Candida-Behandlung und Darmsanierung waren die Hauteffloreszenzen von Traudel D. bereits deutlich abgeklungen, allerdings noch nicht vollständig, bzw. sie blühten immer wieder auf. Ich empfahl ihr daher, mit einem Auslassversuch herauszufinden, ob eine Nahrungsmittelunverträglichkeit bei ihr die Neurodermitis mitverursacht. Verstärken sich die Hautsymptome, sobald ein Nahrungsmittel nach einem mehrwöchigen Verzicht wieder gegessen wird, ist der Auslöser identifiziert, auch ohne aufwendige IgG-Tests. Ich empfahl Traudel D. zunächst auf weizenhaltige Nahrungsmittel zu verzichten. Wie sich herausstellte, war eine Weizenunverträglichkeit bei ihr Mitauslöser der Neurodermitis.

Zusätzlich gab ich der Patientin auch wieder Versandmaterial für eine Stuhluntersuchung mit. Die Stuhlprobe sollte sie jedoch erst 14 Tage vor dem nächsten Termin in meiner Praxis einsenden. 


\section{Beinahe frei von Ekzemen}

Am 19.1.2010 kam Traudel D. wieder in die Sprechstunde. Sie freute sich sehr darüber, dass die Furchen an Händen, Ellenbeugen und Kniekehlen kaum noch sichtbar waren, das Ekzem fast vollständig abgeklungen war, ihre Haut nicht mehr nässte. In der Stuhlprobe war jetzt nur noch eine Kultur mit Candida albicans nachweisbar.

Das Labor sieht diesen Befund als nicht behandlungsbedürftig an. Ich strebe in der Behandlung jedoch eine vollständige Beseitigung des Candida albicans an, denn Rezidive treten meiner Erfahrung nach seltener bei Patienten ohne nachweisbare Kultur auf als bei Patienten mit. Daher riet ich Traudel D. weiterhin zur Einnahme von $3 \times \operatorname{tgl} .1$ Kps. Adiclair ${ }^{\circledR}$ sowie 1 Kps. Mutaflor ${ }^{\circledR} \operatorname{tgl}$. Zur Unterstützung des Heilungsprozesses der Haut verordnete ich zusätzlich $3 \times$ tgl. 1 Tbl. Drüfusan (Fa. Syxyl). Das Präparat beinhaltet die 12 Basissalze nach Dr. Schüßler. Die Gabe potenzierter Mineralstoffe unterstützt den Körper bei der beschleunigten Aufnahme derselben aus der Nahrung und damit respektive bei der Heilung der angegriffenen Haut und Schleimhäute.

Ich gab Traudel D. wiederum Versandröhrchen für eine Stuhluntersuchung mit sowie einen Laborauftrag, wieder sollte sie eine Stuhlprobe erst 14 Tage vor dem nächsten Termin in meiner Praxis zur Untersuchung ins Labor schicken.

\section{Verzicht auf weitere Behandlung}

Als Traudel D. am 2.3.2010 in die Praxis kam, waren die Ekzeme vollständig abgeheilt und ihre Haut frei von Neurodermitis. In der Stuhlprobe war wieder eine Kultur mit Candida albicans nachgewiesen worden. Weil ihre Haut jedoch vollständig frei von Ekzemen war, wollte die Patientin die Behandlung nicht fortsetzen. Ich entließ Traudel D. mit Versandmaterial für eine Stuhluntersuchung und bat sie, dann eine Stuhlprobe an das Labor zu schicken, wenn die Hauteffloreszenzen wieder auftreten.

Bereits im Juni desselben Jahres war das der Fall: Die Neurodermitis war wieder deutlich ausgeprägt und Traudel D. kam erneut in meine Praxis. Der Befund der Stuhluntersuchung lag mir bereits vor, denn sie hatte meiner Weisung entsprechend 14 Tage zuvor eine Stuhlprobe an das Labor gesandt. Wie sich zeigte, war die Darmschleimhaut erneut mit Candida albicans besiedelt, sodass eine Behandlung wieder notwendig war. Ich verordnete wieder $3 \times$ tgl. 2 Kps. Adiclair ${ }^{\circledR}$ sowie $2 \times$ tgl. 1 Kps. Mutaflor ${ }^{\circledR}$. Die Ekzeme heilten innerhalb von 14 Tagen vollständig ab, dieses Mal ohne vorherige Erstverschlimmerung. Bei einer erneuten Stuhluntersuchung war kein Candida albicans mehr nachweisbar.

\section{Rückfall ohne Entmutigung}

Traudel D. musste ihren Beruf nicht aufgeben, sie arbeitet bis heute als Krankenschwester und kann sogar wieder weizenhaltige Nahrungsmittel wie Brot, Kuchen etc. in Maßen zu sich nehmen. Spürt sie ein Jucken an der Haut an den Händen, verzichtet sie vorübergehend auf Weizenprodukte und verhindert so einen erneuten Neurodermitis-Schub.

Traudel D. kam Anfang 2014 noch einmal mit einem Neurodermitis-Schub in meine Praxis. Trotz des vollständigen Verzichts auf Weizenprodukte hatten sich die Hauteffloreszenzen nicht wieder zurückgebildet. Eine Stuhluntersuchung zeigte, dass der Darm wiederum mit Candida albicans besiedelt war. Nach einer kurzfristigen Behandlung mit Adiclair ${ }^{\circledR}, 3 \times$ tgl. 1,5 ml Suspension sowie $3 \times$ tgl. $1 \mathrm{Kps}$., verschwanden die Hauteffloreszenzen wieder vollständig. Bei einer erneuten Stuhluntersuchung waren keine Kulturen mit dem Hefepilz mehr nachweisbar.

\section{Fazit}

Bei einer Besiedelung des Darmes mit Candida albicans lohnt es sich, die Mikrobiologische Therapie so lange fortzuführen, bis keine Kultur mehr positiv ist. Ich empfehle nach Abschluss der Behandlung 2 weitere Kontrolluntersuchungen des Stuhles jeweils im Abstand von 3 Monaten, denn es besteht wie bei allen Pilzerkrankungen ein hohes Rezidivrisiko. Ein Rezidiv sollte jedoch nicht entmutigen. Gerade wenn die Candida-Besiedelung über viele Jahre bestanden hatte, muss eine Mikrobiologische Therapie ggf. mehrfach wiederholt werden. Und das sollte konsequent geschehen, denn nur dann ist eine Behandlung sinnvoll und der Patient auf Dauer ohne Pilzbefall.

Dieser Artikel ist online zu finden:

http://dx.doi.org/10.1055/s-0034-1396952

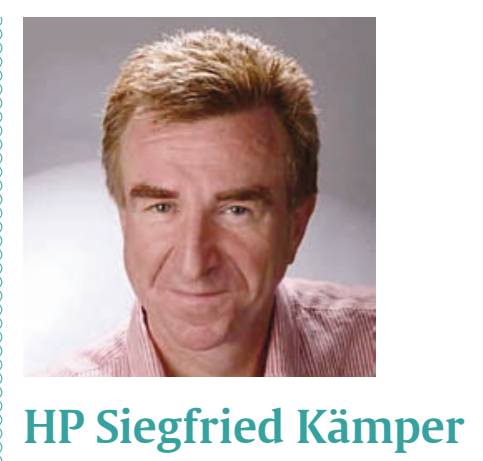

\section{Am Stadtgarten 2 45883 Gelsenkirchen E-Mail: praxis@kaemper.info.de}

Siegfried Kämper ist seit 1985 in eigener Praxis in Gelsenkirchen niedergelassen. Seine Therapie- und Interessensschwerpunkte sind: Mikrobiologische Therapie, Ozontherapie, Chiropraktik, Heilhypnose, Versicherungs- und Abrechnungsfragen, Qualitätssicherung/ Qualitätsmanagement, Arznei- und Medizinprodukterecht. Seit 1987 ist er im Vorstand der Heilpraktikergesellschaft für Ozontherapie e. V., seit 1992 Vizepräsident des BDH. Er ist Leiter der Arzneimittel- sowie der Gutachten- und Gebührenkommission des BDH. Seit 2006 ist er Herausgeber der DHZ. Sein Buch „Praxishandbuch für Heilpraktiker. Abrechnung, Praxisführung, Recht und Hygiene“ ist in der 2. Auflage beim Haug-Verlag erschienen. 